\title{
Comparison of Surgical Skills in Laparoscopic and Robotic Tasks Between Experienced Surgeons and Novices in Laparoscopic Surgery: An Experimental Study
}

\author{
Hye Jin Kim, Gyu-Seog Choi, Jun Seok Park, Soo Yeun Park \\ Colorectal Cancer Center, Kyungpook National University Medical Center, Kyungpook National University School of Medicine, Daegu, Korea
}

Purpose: Robotic surgery is known to provide an improved technical ability as compared to laparoscopic surgery. We aimed to compare the efficiency of surgical skills by performing the same experimental tasks using both laparoscopic and robotic systems in an attempt to determine if a robotic system has an advantage over laparoscopic system.

Methods: Twenty participants without any robotic experience, 10 laparoscopic novices (LN: medical students) and 10 laparoscopically-experienced surgeons (LE: surgical trainees and fellows), performed 3 laparoscopic and robotic training-boxbased tasks. This entire set of tasks was performed twice.

Results: Compared with LN, LEs showed significantly better performances in all laparoscopic tasks and in robotic task 3 during the 2 trials. Within the LN group, better performances were shown in all robotic tasks compared with the same laparoscopic tasks. However, in the LE group, compared with the same laparoscopic tasks, significantly better performance was seen only in robotic task 1 . When we compared the 2 sets of trials, in the second trial, LN showed better performances in laparoscopic task 2 and robotic task 3; LE showed significantly better performance only in robotic task 3 .

Conclusion: Robotic surgery had better performance than laparoscopic surgery in all tasks during the two trials. However, these results were more noticeable for LN. These results suggest that robotic surgery can be easily learned without laparoscopic experience because of its technical advantages. However, further experimental trials are needed to investigate the advantages of robotic surgery in more detail.

Keywords: Robotic surgery; Laparoscopic surgery; Surgical skills

\section{INTRODUCTION}

During the last decade, advances in laparoscopic surgery have expanded its use to various surgical fields, and laparoscopic procedures are now routinely employed to treat diseases. Several randomized trials have shown that laparoscopic surgery is associated

Received: March 12,2013 Accepted: July 17, 2013

Correspondence to: Gyu-Seog Choi, M.D.

Colorectal Cancer Center, Kyungpook National University Medical Center,

130 Dongdeok-ro, Jung-gu, Daegu 700-721, Korea

Tel: +82-53-200-2166, Fax: +82-53-200-2027

E-mail:kyuschoi@mail.knu.ac.kr

(c) 2014 The Korean Society of Coloproctology

This is an open-access article distributed under the terms of the Creative Commons Attribution NonCommercial License (http://creativecommons.org/licenses/by-nc/3.0) which permits unrestricted noncommercial use, distribution, and reproduction in any medium, provided the original work is properly cited. with less surgical trauma, a shorter recovery time and hospital stay, and an improved quality of life [1-4]. However, despite these advantages, laparoscopic procedures are technically demanding for surgeons, owing to several inevitable drawbacks, including twodimensional imaging, an unstable camera platform, limited degrees of freedom due to the rigid instrument, and poor ergonomics [5-8]. For surgical beginners, these factors might contribute to a relatively longer training period in laparoscopy than in open surgery [9].

Surgical robots were introduced in clinical practice in the late 1990s. They were expected to overcome the laparoscopic drawbacks and allow the extension of minimally-invasive surgery to more patients on the basis of technical improvements, such as a three-dimensional view, improved dexterity with an increased range of motion at the tips of the instruments, reduced tremor, enhanced ergonomics, and a stable camera view [10-12]. Compared 


\section{Annals of \\ Coloproctology Hye Jin Kim, et al.}

Comparison of Surgical Skills in Laparoscopic and Robotic Tasks Between Experienced Surgeons and Novices in Laparoscopic Surgery: An Experimental Study

with the conventional laparoscopic approach, robotic systems may also be expected to allow complex laparoscopic tasks to be performed faster and more accurately and to shorten the learning curve $[13,14]$. However, only a few studies have analyzed the superiority of robotic systems, in comparison to laparoscopy, in performing the same procedures $[15,16]$. Recently, a few studies have suggested that robotic systems may offer no advantages over laparoscopic instruments [17, 18].

The laboratory has generally been used to measure the acquisition of surgical skills with different modalities $[19,20]$. Performance in the laboratory can reflect actual surgical procedures within the operating room. It also allows for exact surveillance, regardless of any differences in conditions between the participants, because surgical performance in the operating room does not rely only on technical skills.

Therefore, we compared the efficiencies of surgical skills by using both laparoscopic and robotic systems to perform the same experimental tasks in an attempt to determine the advantages of robotic systems over laparoscopy. We also supposed that the degree of benefits gained from robotic surgery would be different according to laparoscopic skill level because robotic systems are thought to improve surgical performances more quickly than laparoscopic procedures. Therefore, we also compared the differences in skill acquisitions in each surgical modality between novices and surgeons experienced with laparoscopic systems.

\section{METHODS}

This study was approved by the Institutional Review Board at the Kyungpook National University Medical Center, and informed consent was obtained from each participant prior to initiating the study. The 10 laparoscopic novices (LN), consisting of medical students, and 10 LE laparoscopically-experienced surgeons (LE), including surgical residents and fellows, participated in this study. LE had formal education in laparoscopy and had participated in lapa- roscopic surgeries, with more than 3 cases per week and 12 cases per month, but had no experience with robotic systems. LN had no experience with laparoscopic or robotic systems. Before the test, the participants were instructed, through verbal explanations, on the use of both laparoscopic and robotic systems. The participants were allowed two minutes to familiarize themselves with the laparoscopic and the robotic instruments. However, the participants were not allowed to perform any part of the task during the orientation period.

The laparoscopic platform consisted of a training-box, with three preset port sites. A $0^{\circ}$ endoscope (Stryker, San Jose, CA, USA), which was manually controlled by an assistant, was used in the middle port site for visualization. For the performance of the laparoscopic tasks, images were projected onto a 21-inch monitor that was in direct view of the participant (Fig. 1A). Two disposable 5-mm Endo-graspers were used in task 1, 2 disposable 5-mm Endo-dissectors were used in task 2, and a 5-mm Endo-needle holder and disposable 5-mm Endo-dissectors were used in task 3:

\section{Task 1 (pick up and give over a bead)}

Five beads were placed at the bottom of a $5-\mathrm{cm}-\times 5-\mathrm{cm}$-sized circle. Another empty circle was positioned $15 \mathrm{~cm}$ away from the initial circle. The goal of the task was to pick up and transfer 5 beads into the other circle, 1 bead at a time. The participants initially performed the task with their dominant hands and then with their nondominant hands. The 5 repetitions consisted of 5-bead transfers with each hand. The time required for task completion was recorded.

\section{Task 2 (ring insertion onto a cone)}

The participants picked up a rubber ring, transferred it $10 \mathrm{~cm}$ away, and inserted it onto the cone (Fig. 2A). This task was performed with their dominant hand and then with their nondominant hand. For 2 minutes, the total number of inserted rings was recorded.
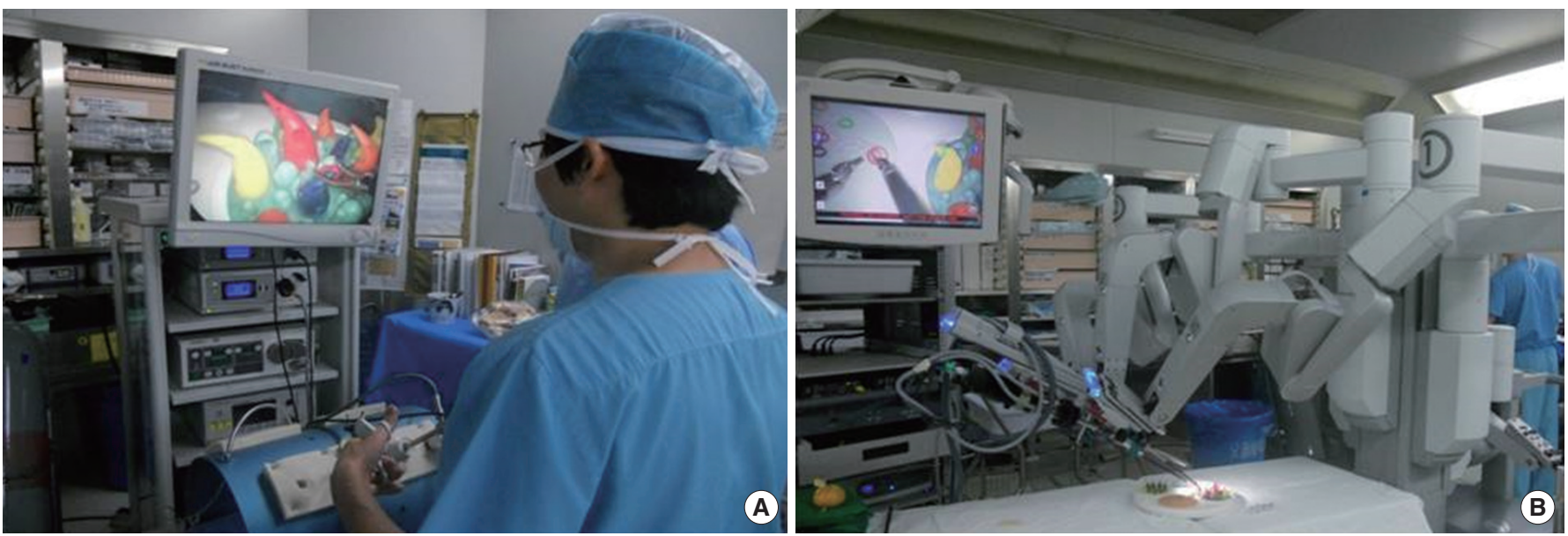

Fig. 1. Laparoscopic platform (A) and robotic platform (B). 


\section{Task 3 (suturing and tying of 3 knots)}

The suturing and tying of 3 knots were evaluated in the training box by using a sponge-block. The task began when the surgeon had loaded the needle, along with a $15-\mathrm{cm}$ polyglactin $3-0$ suture (Vicryl), onto the Endo-needle holder or robotic needle driver (Fig. 2B). An artificial suture sponge was affixed to the floor of a training box, and each person made a suture and tied 3 knots.

The robotic platform consisted of the da Vinci Surgical System (Intuitive Surgical Inc., Sunnyvale, CA, USA), a surgeon's console, three robotic arms, and a vision cart. The participants were seated at the console with a combination of hand and foot controls for the robotic arms, instruments, and an Intuitive $0^{\circ}$ endoscope, which was held by the center arm (Fig. 1B). Three-dimensional images were displayed on the surgeon's console, and a two-dimensional image was projected onto a standard 21-inch monitor for the observer. For all tasks, the EndoWrist instruments, including a needle driver and forceps, were used. Two trials were performed with an 8 -week interval to minimize the effect of the participants' memo- ries of each task.

Statistical calculations were completed using statistical software SPSS ver. 17.0 (SPSS Inc., Chicago, IL, USA). Continuous data between the groups were recorded as the mean value of each variable. Categorical variables were recorded as a number and were compared using the Fisher exact test or $\chi^{2}$ test, as appropriate. A Pvalue of $<0.05$ was considered statistically significant.

\section{RESULTS}

A total of 20 participants completed the tasks on all 3 of the instrument sets. LE consisted of 6 men and 4 women, and the mean age was 30.9 years (range, 28 to 33 years). LN consisted of 5 men and 5 women, and the mean age was 28.2 years (range, 25 to 33 years).

The performance results from all tasks are summarized according to the laparoscopic experience levels in Table 1. Compared to LN, LE showed significantly better performances in all laparoscopic tasks. These results were also shown in the second trial.
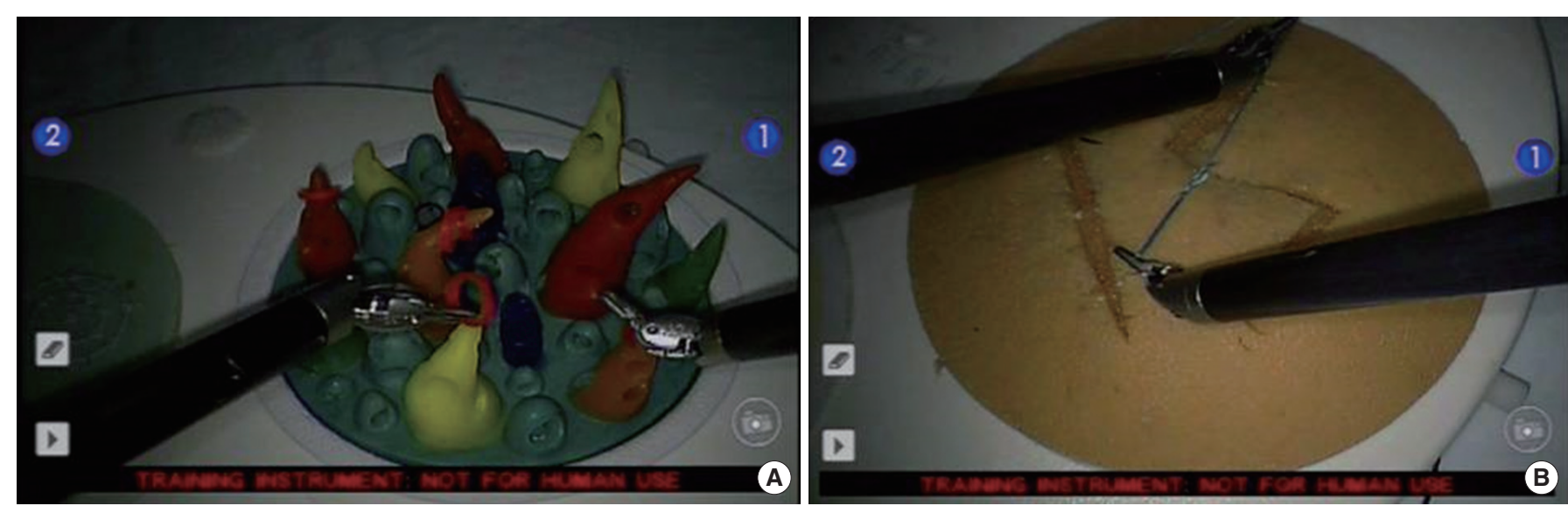

Fig. 2. (A) Robotic task 2, moving the rubber ring and inserting it into the cone, and (B) robotic task 3, suturing and tying three knots.

Table 1. Summary of task results

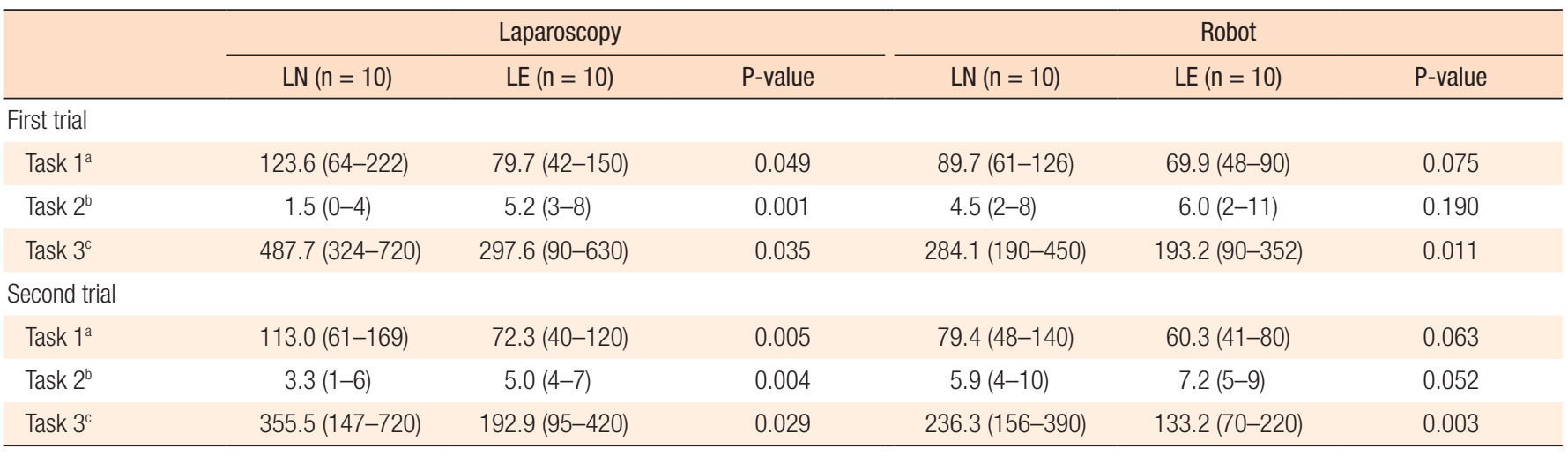

Values are presented as a mean (range).

LN, laparoscopic novice; LE, laparoscopically-experienced surgeon.

TTime (sec) for performing task 1, picking up and giving over the five beads into the circle. ${ }^{b}$ Numbers of rubber ring for performing task 2, moving the rubber ring and inserting it into the cone for 2 minutes. ${ }^{\circ}$ Time (sec) for performing the task 3 , suturing and tying three knots. 
Similarly, in the robotic tasks, LE were faster and demonstrated better performances than LN. However, the only result that was statistically significant in both trials was the measure from robotic task 3 .

The results are summarized according to the surgical modalities

Table 2. Comparison of the times to perform laparoscopic and robotic tasks in all participants

\begin{tabular}{cccr}
\hline & Laparoscopy $(\mathrm{n}=20)$ & Robot $(\mathrm{n}=20)$ & P-value \\
\hline First trial & & & \\
Task 1 & $101.7(42-222)$ & $79.7(48-126)$ & 0.026 \\
Task 2 & $3.4(0-8)$ & $5.3(2-11)$ & 0.039 \\
Task 3 & $392.7(90-720)$ & $238.7(90-450)$ & $<0.001$ \\
Second trial & & & \\
Task 1 & $92.7(40-169)$ & $69.5(48-140)$ & 0.012 \\
Task 2 & $4.2(1-7)$ & $6.6(4-10)$ & 0.024 \\
Task 3 & $274.2(95-720)$ & $184.8(70-330)$ & $<0.001$ \\
\hline
\end{tabular}

Values are presented as a mean (range). in Tables 2 and 3. When comparing all participants regardless of laparoscopic experience level, the robotic system was significantly faster and showed better performance than laparoscopy in all tasks and trials (Table 2). Comparing the two modalities, according to laparoscopic experience levels, within the LN group, better performances were shown in all robotic tasks than in the same laparoscopic tasks. Whereas within the LE group, significantly better performance was observed only in robotic task 1. Comparing the two trials, there was no significant differences except laparoscopic task 2 and robotic task 3 (Table 4).

\section{DISCUSSION}

Advances in laparoscopic surgery have resulted in many changes to the clinical practice of surgery. However, the pitfalls of laparoscopic surgery have still caused undesirable fatigue for surgeons and require relatively longer learning periods than open surgery [9]. When the da Vinci surgical system (Intuitive Surgical Inc.) was introduced for surgery, this robotic approach was expected to offer potential solutions to the problems of the laparoscopic approach in

Table 3. Comparison of the times to perform laparoscopic and robotic tasks according to the experience level of the surgeon with laparoscopy

\begin{tabular}{|c|c|c|c|c|c|c|}
\hline & \multicolumn{3}{|c|}{$\mathrm{LN}(\mathrm{n}=10)$} & \multicolumn{3}{|c|}{$\operatorname{LE}(n=10)$} \\
\hline & Laparoscopy & Robot & P-value & Laparoscopy & Robot & P-value \\
\hline \multicolumn{7}{|l|}{ First trial } \\
\hline Task 1 & 123.6 (64-222) & $89.7(61-126)$ & 0.002 & $79.7(42-150)$ & 69.9 (48-90) & 0.045 \\
\hline Task 2 & $1.5(0-4)$ & $4.5(2-8)$ & 0.002 & $5.2(3-8)$ & $6.0(2-11)$ & 0.529 \\
\hline Task 3 & $487.7(324-720)$ & $284.1(190-450)$ & $<0.001$ & $297.6(90-630)$ & $193.2(90-352)$ & 0.236 \\
\hline \multicolumn{7}{|c|}{ Second trial } \\
\hline Task 1 & $113.0(61-169)$ & $79.4(48-140)$ & 0.002 & $72.3(40-120)$ & $60.3(41-80)$ & 0.043 \\
\hline Task 2 & $3.3(1-6)$ & $5.9(4-10)$ & 0.022 & $5.0(4-7)$ & $7.2(5-9)$ & 0.061 \\
\hline Task 3 & 355.5 (147-720) & 236.3 (156-390) & 0.048 & $192.9(95-420)$ & $133.2(70-220)$ & 0.315 \\
\hline
\end{tabular}

Values are presented as a mean (range).

LN, laparoscopic novice; LE, laparoscopically-experienced surgeon.

Table 4. Comparison of the times to perform the first and the second trials

\begin{tabular}{|c|c|c|c|c|c|c|}
\hline & \multicolumn{3}{|c|}{ Laparoscopy } & \multicolumn{3}{|c|}{ Robot } \\
\hline & First trial & Second trial & P-value & First trial & Second trial & P-value \\
\hline \multicolumn{7}{|c|}{$\operatorname{LN}(n=10)$} \\
\hline Task 1 & 123.6 (64-222) & $113(61-169)$ & 0.853 & $89.7(61-126)$ & $79.4(48-140)$ & 0.315 \\
\hline Task 2 & $1.5(0-4)$ & $3.3(1-6)$ & 0.015 & $4.5(2-8)$ & $5.9(4-10)$ & 0.105 \\
\hline Task 3 & $487.7(324-720)$ & $355.5(147-720)$ & 0.052 & $284.1(190-450)$ & $236.3(156-390)$ & 0.046 \\
\hline \multicolumn{7}{|c|}{$\operatorname{LE}(n=10)$} \\
\hline Task 1 & $79.7(42-150)$ & $72.3(40-120)$ & 0.241 & 59.7 (31-96) & $47.6(18-60)$ & 0.061 \\
\hline Task 2 & $5.2(3-8)$ & $5.0(4-7)$ & 0.062 & $6.0(2-11)$ & $7.2(5-9)$ & 0.253 \\
\hline Task 3 & $297.6(90-630)$ & $192.9(95-420)$ & 0.821 & $193.2(90-352)$ & $133.2(70-220)$ & 0.049 \\
\hline
\end{tabular}

Values are presented as a mean (range).

LN, laparoscopic novice; LE, laparoscopically-experienced surgeon. 
various surgical fields based on the advancement of technology [8$10,21]$. However, to date, only a few studies have analyzed the advantages of robotic systems in comparison to laparoscopic approaches by measuring the performances of the same procedures $[15,16]$. Therefore, in the present study, we compared the surgical skill acquisition between conventional laparoscopic instruments and surgical robotic systems through the experimental performance of basic laparoscopic tasks to demonstrate the advantages of robotic systems and laparoscopic instruments.

In the present study, the participants had significantly better results with the robotic system than with the laparoscopic instruments while performing the same basic skills. These results originated from the several previously-mentioned advantages of robotic systems over conventional laparoscopic instruments. Among them, improved dexterity, with multiarticulated instruments mimicking the human wrist, and high-definition three-dimensional view may mainly contribute to the rapid skill acquisition when using robotic system. First, the EndoWrist instruments of the robotic system especially contributed to the excellent records in performing task 3, compared to laparoscopic approach, because the articulating robotic needle driver is very convenient for suturing and tying a knot. As a result, LN could shorten the amount of time to suture and tie a knot 3 times by up to 200 seconds in the first trial and by up to 120 seconds in the second trial (Table 3). LE also shortened the actual time of performing task 3 by up to $100 \mathrm{sec}-$ onds in the first trial and by up to 60 seconds in the second trial, although the time differences among the 2 approaches were not statistically significant. The three-dimensional view improved the participants' visual perspectives and precise movement abilities in picking up and placing the object by recovering the perspectives. In addition, the robotic system could help the participants perform basic tasks with their unskilled, nondominant hands when they were required to alternate and coordinate their dominant and nondominant hands in the robotic console. Because of this strength of the robotic system, the participants were had the time necessary to accurately and precisely perform all of the tasks (Table 3).

However, the robotic system seems to have given more benefits to the LN group than the LE group. The reason is that the LN group had significantly better performances with the robotic systems in all tasks than they had with the laparoscopic instruments, but LE only had statistically significant better results in robotic task 1 of the 2 trials compared to that of the laparoscopic tasks. However, all LE also had better results for the robotic tasks than they had for the laparoscopic tasks. Laparoscopic experience may contribute to the better performance results seen in the robotic tasks, considering the better performance results of LE. However, based on the results of the LN, the surgeon who does not have any surgical or laparoscopic experience also can rapidly learn to perform surgical tasks in less time by using robotic systems. One possible reason for these results may be that robotic systems have a more user-friendly interface than laparoscopic instruments do [16]. Another explanation might be that robotic systems are designed spe- cifically to mimic the same hand motions as those used during open surgery. We deem that our results support the concept that the inexperienced in laparoscopic surgery may have improved proficiencies and may more quickly and easily acquire surgical skills with the use of robotic systems.

In the present study, although there were only two trials, by using both the laparoscopic and the robotic systems, the participants seemed to show more improvements in speed and skill with repetition in performing robotic tasks. Although this result did not represent an improvement of surgical skill, it shows the possibility of a shorter learning period in the robotic approach because the improvement in the result was noticeable in robotic surgery, regardless of the participants' laparoscopic experience levels and despite the higher score in the first trial compared to laparoscopic approach. The preliminary result of the learning curve for the robotic approach showed that the use of the robotic approach may even reduce the learning curve for the laparoscopic approach. Another recent study showed that the transfer of an open surgical skill to a video-assisted environment required only 12 robotic operations, with outcomes similar to those of a skilled laparoscopic surgeon after more than 100 laparoscopic procedures [14]. However, if a more complete characterization of the learning curve is to be established, experiments, including more extended repetitions, are required.

This study had several limitations. The conventional variables measured during the task performance, time and score, are regarded as being insufficient to fully explain the aspects of surgical performance [22, 23]. Skilled performance with a robotic system may include other qualitative aspects, and these should be addressed in the objective assessment of the performance. In addition, suturing and tying a knot is a procedure to acquire a skill by practicing a method. Therefore, the previous experience with open surgery in the LE group may have the effect of producing a better result in performing task 3 in the LE group than in LN group. A more objective module is required to evaluate skill acquisition according to the surgical approach. Another limitation was the limited number of repetitions in performing the tasks. Therefore, we cannot speculate on the learning curve for robotic systems based on these results.

Skill development in a laparoscopic or robotic system is fundamentally different from that in open surgery; to date, there has been limited research regarding surgical skill acquisition with these sophisticated systems [24, 25]. Robotic systems are still developing, and with more experience and adjusted equipment, performance with robotic systems is likely to improve. More research is required to assess the advantages and the disadvantages of using both laparoscopic and robotic systems. Comparisons regarding performances with robotic systems and with conventional laparoscopic instruments, particularly with more complex tasks, should, therefore, be made after consistent performance baselines have been determined to avoid confounding issues that are more related to the learning curve of each modality. 


\section{CONFLICT OF INTEREST}

No potential conflict of interest relevant to this article was reported.

\section{ACKNOWLEDGMENTS}

This work was supported by a Biomedical Research Institute grant, Kyungpook National University Hospital (2011).

\section{REFERENCES}

1. A prospective analysis of 1,518 laparoscopic cholecystectomies. The Southern Surgeons Club. N Engl J Med 1991;324:1073-8.

2. Lacy AM, Garcia-Valdecasas JC, Delgado S, Castells A, Taura P, Pique JM, et al. Laparoscopy-assisted colectomy versus open colectomy for treatment of non-metastatic colon cancer: a randomised trial. Lancet 2002;359:2224-9.

3. Guillou PJ, Quirke P, Thorpe H, Walker J, Jayne DG, Smith AM, et al. Short-term endpoints of conventional versus laparoscopicassisted surgery in patients with colorectal cancer (MRC CLASICC trial): multicentre, randomised controlled trial. Lancet 2005; 365:1718-26.

4. Korolija D, Sauerland S, Wood-Dauphinee S, Abbou CC, Eypasch E, Caballero MG, et al. Evaluation of quality of life after laparoscopic surgery: evidence-based guidelines of the European Association for Endoscopic Surgery. Surg Endosc 2004;18:879-97.

5. Ballantyne GH. The pitfalls of laparoscopic surgery: challenges for robotics and telerobotic surgery. Surg Laparosc Endosc Percu$\tan$ Tech 2002;12:1-5.

6. Gallagher AG, Richie K, McClure N, McGuigan J. Objective psychomotor skills assessment of experienced, junior, and novice laparoscopists with virtual reality. World J Surg 2001;25:1478-83.

7. Gutt CN, Oniu T, Mehrabi A, Kashfi A, Schemmer P, Buchler MW. Robot-assisted abdominal surgery. Br J Surg 2004;91:1390-7.

8. Quick NE, Gillette JC, Shapiro R, Adrales GL, Gerlach D, Park $\mathrm{AE}$. The effect of using laparoscopic instruments on muscle activation patterns during minimally invasive surgical training procedures. Surg Endosc 2003;17:462-5.

9. Subramonian K, DeSylva S, Bishai P, Thompson P, Muir G. Acquiring surgical skills: a comparative study of open versus laparoscopic surgery. Eur Urol 2004;45:346-51.

10. D’Annibale A, Morpurgo E, Fiscon V, Trevisan P, Sovernigo G, Orsini C, et al. Robotic and laparoscopic surgery for treatment of colorectal diseases. Dis Colon Rectum 2004;47:2162-8.

11. Ballantyne GH. Robotic surgery, telerobotic surgery, telepresence, and telementoring. Review of early clinical results. Surg Endosc 2002;16:1389-402.
12. Cadiere GB, Himpens J, Germay O, Izizaw R, Degueldre M, Vandromme J, et al. Feasibility of robotic laparoscopic surgery: 146 cases. World J Surg 2001;25:1467-77.

13. Garcia-Ruiz A, Gagner M, Miller JH, Steiner CP, Hahn JF. Manual vs robotically assisted laparoscopic surgery in the performance of basic manipulation and suturing tasks. Arch Surg 1998;133: 957-61.

14. Ahlering TE, Skarecky D, Lee D, Clayman RV. Successful transfer of open surgical skills to a laparoscopic environment using a robotic interface: initial experience with laparoscopic radical prostatectomy. J Urol 2003;170:1738-41.

15. Heemskerk J, van Gemert WG, de Vries J, Greve J, Bouvy ND. Learning curves of robot-assisted laparoscopic surgery compared with conventional laparoscopic surgery: an experimental study evaluating skill acquisition of robot-assisted laparoscopic tasks compared with conventional laparoscopic tasks in inexperienced users. Surg Laparosc Endosc Percutan Tech 2007;17:171-4.

16. Yohannes P, Rotariu P, Pinto P, Smith AD, Lee BR. Comparison of robotic versus laparoscopic skills: is there a difference in the learning curve? Urology 2002;60:39-45.

17. Melvin WS, Needleman BJ, Krause KR, Schneider C, Wolf RK, Michler RE, et al. Computer-enhanced robotic telesurgery. Initial experience in foregut surgery. Surg Endosc 2002;16:1790-2.

18. Nio D, Balm R, Maartense S, Guijt M, Bemelman WA. The efficacy of robot-assisted versus conventional laparoscopic vascular anastomoses in an experimental model. Eur J Vasc Endovasc Surg 2004;27:283-6.

19. Kopta JA. An approach to the evaluation of operative skills. Surgery 1971;70:297-303.

20. Reznick R, Regehr G, MacRae H, Martin J, McCulloch W. Testing technical skill via an innovative "bench station" examination. Am J Surg 1997;173:226-30.

21. Choi GS, Park IJ, Kang BM, Lim KH, Jun SH. A novel approach of robotic-assisted anterior resection with transanal or transvaginal retrieval of the specimen for colorectal cancer. Surg Endosc 2009; 23:2831-5.

22. Bann SD, Khan MS, Darzi AW. Measurement of surgical dexterity using motion analysis of simple bench tasks. World J Surg 2003;27: 390-4.

23. Hernandez JD, Bann SD, Munz Y, Moorthy K, Datta V, Martin S, et al. Qualitative and quantitative analysis of the learning curve of a simulated surgical task on the da Vinci system. Surg Endosc 2004; 18:372-8.

24. Hashizume M, Konishi K, Tsutsumi N, Yamaguchi S, Shimabukuro R. A new era of robotic surgery assisted by a computer-enhanced surgical system. Surgery 2002;131(1 Suppl):S330-3.

25. Jacobs LK, Shayani V, Sackier JM. Determination of the learning curve of the AESOP robot. Surg Endosc 1997;11:54-5. 